\title{
Electron Energy Spectra from the Strong-Field Double-Ionization of Xenon
}

\author{
J.L. Chaloupka ${ }^{a}$, R. $\operatorname{Lafon}^{a}$, J. Rudati ${ }^{a}$, B. Sheehy ${ }^{a}$, \\ P. Agostini ${ }^{b}$, K.C. Kulander ${ }^{c}$ And L.F. DiMauro ${ }^{a}$ \\ ${ }^{a}$ Brookhaven National Laboratory, Upton NY 11973, USA \\ ${ }^{b}$ SPAM, Centre d'Etudes de Saclay, 91191 Gif sur Yvette, France
}

${ }^{\circ}$ TAMP, Lawrence Livermore National Laboratory, Livermore, CA 94551, U.S.A.

\begin{abstract}
The electron energy spectra correlated to the strong-field double-ionization of xenon are presented at three laser intensities. The double-ionization electrons are on average more energetic than those generated from single-ionization. This difference in energy is not manifested as a simple scaling to higher electron energies, but rather as a change in the shape of the spectra. This trend is observed at all intensities. Most notably, the comparison between single- and double-ionization spectra is very similar at low and high peak laser intensities. This could imply that a sequential double-ionization process dominates at all intensities, even where the double ion yield is enhanced.
\end{abstract}

PACS numbers: $32.80 . \mathrm{Fb}, 31.90 .+\mathrm{s}, 32.80 . \mathrm{Rm}$

\section{Introduction}

Soon after the discovery of the laser in the early 1960 s, a fundamental phenomenon known as multi-photon ionization (MPI) was observed by Voronov and Delone [1]. Using the intense visible light generated by a $Q$-switched ruby laser, they were able to ionize xenon atoms. This was significant in that the individual photon energy of the laser light was much lower than the ionization potential of xenon. In contrast to ordinary photoionization, where a single photon of sufficient energy liberates an electron from the atom, MPI can occur for arbitrarily low photon energies provided that the intensity of the light is high enough. In the case of Voronov and Delone, seven $1.78 \mathrm{eV}$ photons conspire together to release a single electron bound by $12.13 \mathrm{eV}$. 
Since that time, the study of MPI in particular and of strong-field laser-atom interactions in general has been an active discipline in atomic physics. The ionization of atoms with an excess number of photons (beyond what is minimally required) was observed and dubbed above-threshold ionization [2] in 1979. Multiple MPI has also been observed, where more than one electron is liberated by the intense field. First, in 1977 in alkali earth metals [3], and in 1982 in a rare gas [4]. Since then, the ion yields for single and multiple ionization have been meticulously recorded [5-7]. The electron energy spectra resulting from single-ionization have also been observed [8-11]. From these experiments, a framework was developed that successfully described the process of strong-field, single-ionization [12-16]. However, the double-ionization process could not be modeled simply as an extension of single-ionization. While the assumption that single-ionization occurred with only one "active" electron led to good agreement with experiment, this was not the case with double-ionization. Most notably, the double ion yields in helium were dramatically enhanced from the single-active-electron models at low intensities $[5,6]$, leading to a number of theoretical explanations for the discrepancy [17-28]. Each of these descriptions incorporated some sort of "non-sequential" process, where the liberation of the second electron did not occur in an independent manner following the release of the first electron.

It became clear that in order to understand the double-ionization process, measurements in the laboratory would have to distinguish between single- and double-ionization beyond the accumulation of ion yields. Very recently, experimental advances have led to just such techniques [29-37]. Through the use of cold target recoil ion momentum spectroscopy (COLTRIMS), the ion recoil momenta of argon [29], neon [30], and helium [31] double-ionization have been measured, along with the electron momenta in argon [32-34]. Electron-ion coincidence spectroscopy has been used to measure the energy spectra of electrons correlated to double-ionization in xenon [35], helium [36] and the rare gases spanning He to $\mathrm{Xe}$ [37]. In this article, we present new electron energy spectra resulting from the single- and double-ionization of xenon. These results, taken at three intensities and with high statistics, show features reminiscent of tunneling ionization as well as MPI. Due to this ambiguous placement within the regimes of strong-field ionization, conclusions about the nature of the double-ionization process are much less clear than in the purely tunneling case of helium. Nevertheless, indications exist that the double-ionization of xenon could have a "sequential" nature even in a low, a priori "non-sequential" intensity range. We also present electron energy spectra correlated to the production of $\mathrm{Xe}^{+}$through $\mathrm{Xe}^{3+}$ from a third, higher intensity.

\section{Experimental scheme}

In order to compile the electron energy spectrum correlated to the creation of a particular ion, it is necessary to simultaneously detect the ions and electrons 
generated for each individual laser shot. By keeping the count rate relatively low, one can be reasonably certain that the detected ion and electron(s) were generated from the same ionization process. The probability that the ion and electron are or are not positively correlated is calculable [38] and depends on the electron and ion detection efficiencies, the ionization rates of the various ion species and the total ion detection rate. These probabilities can be expressed as a ratio of true to false ( $\mathrm{T}: \mathrm{F}$ ) counts. As can be expected, better detection efficiencies will yield higher T:F values. In the ideal case, a perfect ion detector would result in identically zero false counts. In such an extreme (and unrealistic) situation, any laser shot where more than one ionization event takes place would be immediately discarded. Only those instances where exactly one ionization event occurs would be considered, leaving no room for a false coincidence event. For realistic experimental conditions (i.e., imperfect detectors), the $\mathrm{T}: \mathrm{F}$ ratio can be controlled by varying the total count rate. The T:F ratio can be made arbitrarily high even for poor detection efficiencies by sufficiently lowering the count rate. By doing this, the likelihood of even generating more than one ion/electron pair is minimized, greatly reducing the odds of detecting an uncorrelated ion and electron. This has the distinct advantage of increasing the certainty of positive electron-ion correlation, but the severe disadvantage of reducing the rate of data accumulation, and inevitably resulting in a reduction in statistics. The experimentalist must find a reasonable "middle ground" between poor statistics/high T:F and good statistics/low T:F. In these studies, a total detection rate of $25 \%$ was used, leading to typical double-ionization $\mathrm{T}: \mathrm{F}$ ratios of roughly $4: 1$. Since the "false" spectrum is always known (equal to the total, non-coincident electron spectrum), it is possible to subtract away the contribution of false counts. For the results presented here, these corrections make no qualitative change and are therefore not presented.

A two-sided, pulsed-plate spectrometer was used to detect the time-of-flight of electrons and the charge-to-mass ratio of ions generated in the ionization process $[35,36]$. The $100-\mathrm{fs}, 780-\mathrm{nm}$ pulses from a $2-\mathrm{kHz}$ repetition rate, regeneratively amplified Ti:sapphire laser were tightly focused in the center of the spectrometer interaction region inside an ultrahigh vacuum chamber. The chamber has a base pressure of $10^{-10}$ torr and is backfilled with xenon gas at pressures ranging from $10^{-9}$ to $10^{-6}$ torr depending on the laser intensity and the desired count rate. The interaction region is initially grounded and field-free, allowing for the unimpeded and natural drift of the electrons away from the laser focus. Only those electrons traveling towards the detector, along the laser polarization direction and within a $10^{\circ}$ acceptance angle can be detected. The majority of the $20-\mathrm{cm}$ distance to the electron detector is grounded and shielded from the interaction region. Therefore, after a short delay, it is possible to apply an extraction field to pull the ions out of the interaction region without affecting the drift of the electrons. This two-sided scheme, where the ion and electron spectrometers are located perpendicular to the laser propagation direction on opposite sides of the focus, allows for the in- 
dependent optimization of both electron and ion detection. Experimental tests showed that both the electron and ion spectrometers imaged the same region in the interaction region. The ion and electron collection and detection efficiencies are roughly $30 \%$ and $1 \%$, respectively. For an experimental run of $10^{6}$ laser shots, the total electron and ion time-of-flight spectra are recorded. In addition, the ion and electron arrival times are recorded whenever precisely one ion is detected. With this information, it is possible to compile the electron energy spectrum correlated to the detection of any given ion species and charge state. The results of a controlled test of the spectrometer and the coincidence scheme have been presented elsewhere [36].

\section{Experimental results}

The studies discussed here were carried out at three peak laser intensities. In order to determine the intensity regions of interest, the single- and double-ionization yields were measured at various laser powers. The laser intensity was calibrated through the use of the well-known helium photoelectron energy spectrum [10, 11]. In helium, single-ionization has been successfully described as the tunneling of the electron through the suppressed Coulomb barrier. In such a description, the electron is most likely to gain zero energy from the laser field, but can possibly gain as much as twice its quiver energy. This cycle-averaged energy is known as the ponderomotive potential $\left(U_{\mathrm{p}}\right)$ and scales linearly with intensity. In addition to the zero to $2 U_{\mathrm{p}}$ range of allowed final energies, there is a small probability that the electron can gain as much as $10 U_{\mathrm{p}}$ from the field through a rescattering process with the ion. As a result, the photoelectron spectrum in the tunneling regime exhibits a sharp drop-off towards $2 U_{\mathrm{p}}$, followed by an extended plateau towards $10 U_{\mathrm{p}}$. This break in slope at the $2 U_{\mathrm{p}}$ point can be used to determine the peak laser intensity.

The $\mathrm{Xe}^{2+}: \mathrm{Xe}^{+}$ion yield ratios are plotted in Fig. 1 (circles). The gray line shows a ratio curve calculated with the single- and double-ionization saturation intensities at $I_{1}$ and $I_{2}$, respectively. The saturation intensity is defined as the highest intensity that the atom can experience before being ionized with certainty. The calculated curve is not intended to match the observed ratio, but rather to show the appropriate labeling of the saturation intensities. Beyond the double-ionization saturation intensity $\left(I_{2}\right)$, one might expect the curve to be completely level, since the single- and double-ionization focal volumes should grow as the intensity to the $3 / 2$ power. This $I^{3 / 2}$ dependence is correct, but not at very small focal volumes. The initial growth in both the calculated and observed ratio values beyond the double-ionization saturation intensity is a result of this small focal volume behavior. Eventually, the ratio values reach the high intensity limit of $\left(I_{2} / I_{1}\right)^{3 / 2}$ as expected.

At $8 \times 10^{13} \mathrm{~W} / \mathrm{cm}^{2}$ (labeled $I_{1}$ ), there is a clear "bump" in the ratio curve. A similar feature is seen in all of the rare gases but most dramatically in he- 


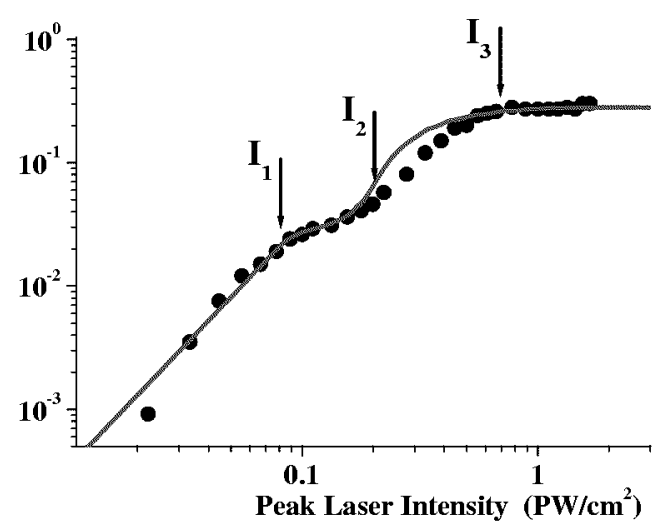

Fig. 1. The ratio of $\mathrm{Xe}^{2+}$ production with respect to $\mathrm{Xe}^{+}$is shown as a function of peak laser intensity. The three intensities at which the electron-ion coincidence studies were performed are labeled. Note the bump in the ratio curve at $I_{1}$, resulting from an enhanced double-ionization rate.

lium [6], where the enhancement of the double-ionization rate is attributed to a non-sequential process [15]. If such a process also occurs in xenon, it should be evident from the electron energy spectra collected at this intensity. At the second intensity $\left(I_{2}=2 \times 10^{14} \mathrm{~W} / \mathrm{cm}^{2}\right)$, double-ionization is no longer dominated by the "enhanced" rate. If the double-ionization process is dictated by two different mechanisms at $I_{1}$ and $I_{2}$, then this should be reflected by a change in the structure of the double-ionization electron spectra. Such behavior has been previously observed in ion recoil momentum measurements in argon [29] and helium [31] and electron measurements in argon [32]. The third intensity $\left(I_{3}=7 \times 10^{14} \mathrm{~W} / \mathrm{cm}^{2}\right)$ allows for the observation of electrons correlated to triple ionization.

Figure 2 shows the electron energy spectra correlated to single- (solid line) and double- (dashed line) ionization of xenon at $8 \times 10^{13} \mathrm{~W} / \mathrm{cm}^{2}\left(I_{1}\right)$. The counts along the vertical axis are normalized for each spectrum such that the total integrated counts equal unity for each curve. The total electron counts equal $5.4 \times 10^{5}$ for single-ionization and $1.3 \times 10^{4}$ for double-ionization. The energies corresponding to $2 U_{\mathrm{p}}$ and $10 U_{\mathrm{p}}$ are marked on the plot. At this intensity the ionization of xenon is dominated by the multiphoton process [8], so the change in slope at $2 U_{\mathrm{p}}$ associated with tunneling is not observed. In the purely tunneling case of helium, the single-ionization spectrum falls rapidly towards $2 U_{\mathrm{p}}$ and then extends in a plateau towards $10 U_{\mathrm{p}}$, while the double-ionization spectrum extends beyond $2 U_{\mathrm{p}}$ with no change in slope. This stark contrast highlights the difference between the tunneling process for single-ionization and the non-sequential process for double-ionization. In fact, the existence of helium double-ionization electrons beyond $2 U_{\mathrm{P}}$ at relatively high rates gives strong evidence for the rescattering scenario [36], where the first electron is driven back by the laser field towards the ion, leading to 


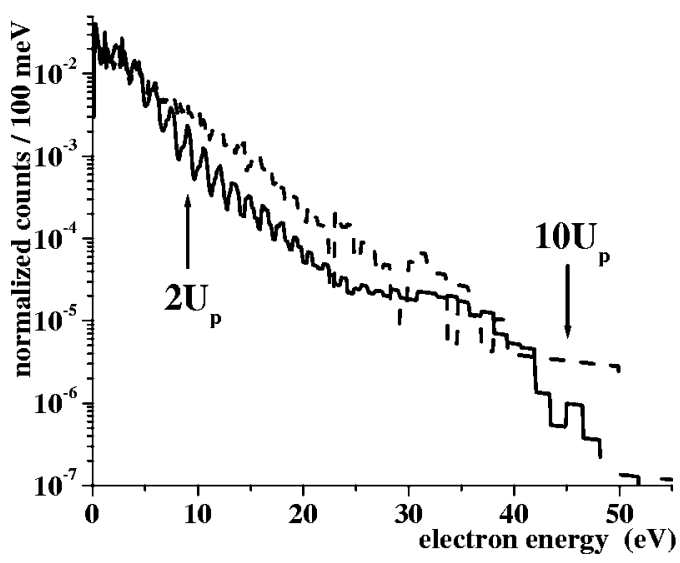

Fig. 2. Electron spectra recorded in coincidence with the detection of a single $\mathrm{Xe}^{+}$ ion (solid line) or a single $\mathrm{Xe}^{2+}$ ion (dashed line) are shown for an intensity of $I_{1}=$ $8 \times 10^{13} \mathrm{~W} / \mathrm{cm}^{2}$. The $2 U_{\mathrm{p}}$ and $10 U_{\mathrm{p}}$ points corresponding to this intensity are shown.

impact ionization [15]. Here, in the case of xenon, such a strong conclusion cannot be made.

Figure 3 shows the single- (solid line, $5.0 \times 10^{5}$ counts) and double- (dashed line, $3.0 \times 10^{4}$ counts) ionization electron spectra taken at $2 \times 10^{14} \mathrm{~W} / \mathrm{cm}^{2}\left(I_{2}\right)$. One might argue that the spectra begin to exhibit tunneling-like behavior, seen as a break in slope near $2 U_{\mathrm{p}}$, in both cases. However, at this intensity the production of $\mathrm{Xe}^{2+}$ is no longer dominated by an enhanced rate and is therefore considered to be sequential. As a result, the observation of similar electron spectra from single- and

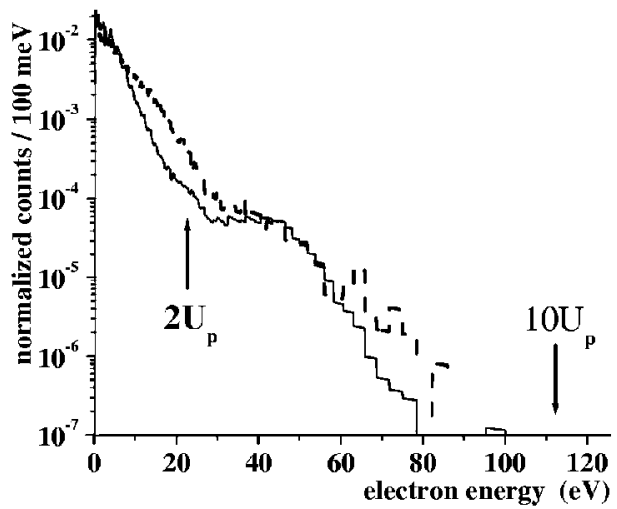

Fig. 3. Electron spectra recorded in coincidence with the detection of a single $\mathrm{Xe}^{+}$ ion (solid line) or a single $\mathrm{Xe}^{2+}$ ion (dashed line) are shown for an intensity of $I_{2}=2 \times 10^{14} \mathrm{~W} / \mathrm{cm}^{2}$. The $2 U_{\mathrm{p}}$ and $10 U_{\mathrm{p}}$ points corresponding to this intensity are shown. 
double-ionization is not surprising. As in Fig. 2, the double-ionization spectrum is somewhat more energetic than the single-ionization case, and the single- and double-ionization curves seem to scale with one another in similar ways. This is unusual considering the location of $I_{1}$ and $I_{2}$ in the ion ratio curve of Fig. 1. In COLTRIMS studies performed in argon [29,32] and helium [31], the distribution of ion or electron momenta showed clear differences between low and high intensities. The fact that the single- and double-ionization electron spectra taken in xenon at $I_{1}$ and $I_{2}$ scale in similar ways implies that there is no dramatic change in the nature of the double-ionization process between these two intensities. Our results here are in good agreement with the only other electron-ion coincidence study performed in xenon [35].

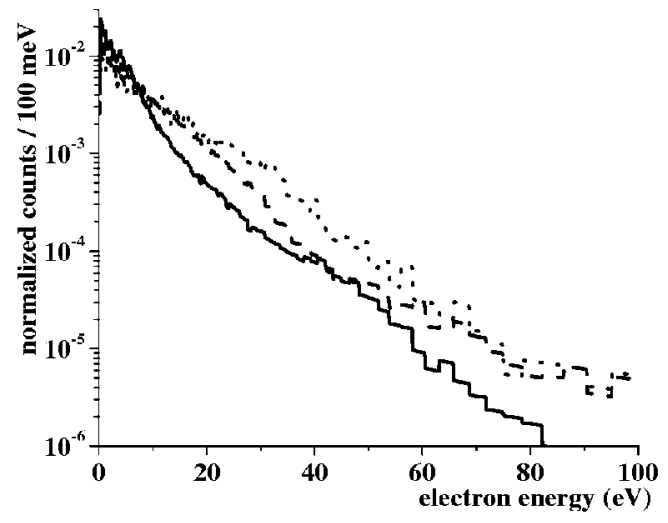

Fig. 4. Electron spectra recorded in coincidence with the detection of a single $\mathrm{Xe}^{+}$ion (solid line) or a single $\mathrm{Xe}^{2+}$ ion (dashed line) or a single $\mathrm{Xe}^{3+}$ ion (dotted line) are shown for an intensity of $I_{3}=7 \times 10^{14} \mathrm{~W} / \mathrm{cm}^{2}$.

Figure 4 shows the electron energy spectra taken at $7 \times 10^{14} \mathrm{~W} / \mathrm{cm}^{2}\left(I_{3}\right)$ correlated to single- (solid line, $1.7 \times 10^{5}$ counts), double- (dashed line, $7.6 \times 10^{4}$ counts) and triple- (dotted line, $1.1 \times 10^{4}$ counts) ionization. The trends observed for the double-ionization spectra at the two lower intensities are observed here and are also seen in the triple-ionization spectrum. The relative rates at low electron energies are smaller than in the single-ionization case, but the drop-off towards high energies is more shallow, leading to enhanced relative rates at higher electron energies. The electrons released through triple-ionization are more energetic than those from double-ionization, which in turn are more energetic than the single-ionization electrons. This is not surprising, since even though the peak laser intensity is the same for all three cases, the saturation intensity differs among them. As a result, the intensity at which a given electron is released into the field will be higher on average for the higher charge states. Although this could explain the difference in average electron energy, it does not address the difference in the 
shapes of the electron spectra. If double- and triple-ionizations occur through the exact same process as single-ionization, then the spectra should simply scale with a saturation intensity. The differences in the shapes of the electron spectra likely point to some interesting complexities in the multiple ionization of xenon.

\section{Conclusions}

We have presented new results in our studies of the strong-field ionization of xenon. Using an electron-ion coincidence technique, the electron spectra correlated to the detection of singly and doubly charged ions have been compiled at two key intensities. In contrast to electron and ion recoil momenta measurements performed in argon and helium, there are no significant changes in the structure of the electron distributions between low and high laser intensities. At the same time, the shapes of the spectra differ beyond a simple scaling factor. It appears that the double-ionization process may not be non-sequential at any intensity, while still differing from the single-ionization case. Since the ionization of xenon is more multiphoton in nature than the previously studied gases, it is possible that the enhancement of the double-ionization rate at low intensities is due to a different process. The effect of atomic resonances leading to a non-monotonic double-ionization yield has been previously discussed [39,40]. This could lead to the tell-tale structure seen in the $\mathrm{Xe}^{2+}: \mathrm{Xe}^{+}$ratio curve within a sequential picture. We have also presented the electron spectra correlated to $\mathrm{Xe}^{+}$through $\mathrm{Xe}^{3+}$ at a higher intensity. Here, the trends between the single- and double-ionization spectra at the lower intensities are confirmed and are mirrored in the triple-ionization spectrum. An on-going study of the xenon double-ionization electron spectra at lower intensities, in particular where the above-threshold ionization and atomic resonance peaks are most visible in the single-ionization spectra, may provide more insight into the double-ionization process.

\section{Acknowledgments}

The experiments were carried out at Brookhaven National Laboratory under contract No. DE-AC02-98CH10886 with the United States Department of Energy and supported by its Division of Chemical Sciences, Office of Basic Energy Sciences.

\section{References}

[1] G.S. Voronov, N.B. Delone, JETP Lett. 1, 66 (1965).

[2] P. Agostini, F. Fabre, G. Mainfray, G. Petite, Phys. Rev. Lett. 42, 1127 (1979).

[3] I.S. Aleksakhin, I.P. Zapesochnyi, V.V.Suran, JETP Lett. 26, 11 (1977). 
[4] A. L'Huillier, L.A. Lompre, G. Mainfray, C. Manus, Phys. Rev. Lett. 48, 1814 (1982).

[5] D.N. Fittinghoff, P.R. Bolton, B. Chang, K.C. Kulander, Phys. Rev. Lett. 69, 2642 (1992).

[6] B. Walker, B. Sheehy, L.F. DiMauro, P. Agostini, K.J. Schafer, K.C. Kulander, Phys. Rev. Lett. 73, 1227 (1994).

[7] S. Larochelle, A. Talebpour, S.L. Chin, J. Phys. B 31, 1201 (1998).

[8] E. Mevel, P. Breger, R. Trainham, G. Petite, P. Agostini, A. Migus, J.-P. Chambaret, A. Antonetti, Phys. Rev. Lett. 70, 406 (1993).

[9] U. Mohideen, M.H. Sher, H.W.K. Tom, G.D. Aumiller, O.R. Wood II, R.R. Freeman, J. Bokor, P.H. Bucksbaum, Phys. Rev. Lett. 71, 509 (1993).

[10] B. Walker, B. Sheehy, K.C. Kulander, L.F. DiMauro, Phys. Rev. Lett. 77, 5031 (1996).

[11] B. Sheehy, R. Lafon, M. Widmer, B. Walker, L.F. DiMauro, P.A. Agostini, K.C. Kulander, Phys. Rev. A 58, 3942 (1998).

[12] M.V. Ammosov, N.B. Delone, V.P. Krainov, Sov. Phys. JETP 64, 1191 (1987).

[13] L.F. DiMauro, P. Agostini, Adv. At. Mol. Opt. Phys. 35, 79 (1995).

[14] A. Becker, L. Plaja, P. Moreno, M. Nurhuda, F.H.M. Faisal, Phys. Rev. A 64, 023408 (2001).

[15] P.B. Corkum, Phys. Rev. Lett. 71, 1994 (1993).

[16] K.J. Schafer, B. Yang, L.F. DiMauro, K.C. Kulander, Phys. Rev. Lett. 70, 1599 (1993).

[17] J.B. Watson, A. Sanpera, D.G. Lappas, P.L. Knight, K. Burnett, Phys. Rev. Lett. 78, 1884 (1997).

[18] J.S. Parker, E.S. Smyth, K.T. Taylor, J. Phys. B 31, L571 (1998).

[19] M.A. Kornberg, P. Lambropoulos, J. Phys. B 32, L603 (1999).

[20] A. Becker, F.H.M. Faisal, Phys. Rev. Lett. 84, 3546 (2000).

[21] R. Panfili, C. Szymanowski, W.-C. Liu, J.H. Eberly, in: Multiphoton Processes, Eds. L.F. DiMauro, R.R. Freeman, K.C. Kulander, American Institute of Physics, New York (NY) 2000, p. 265.

[22] M. Lein, E.K.U. Gross, V. Engel, Phys. Rev. Lett. 85, 4707 (2000).

[23] G.L. Yudin, M. Ivanov, Phys. Rev. A 63, 033404 (2001).

[24] U. Eichmann, M.Dörr, H. Maeda, W. Becker, W. Sandner, Phys. Rev. Lett. 84, 3550 (2000).

[25] S.V. Popruzhenko, S.P. Goreslavskii, J. Phys. B 34, L239 (2001).

[26] K. Sacha, B. Eckhardt, Phys. Rev. A 63, 043414 (2001).

[27] N.E. Dahlen, R. van Leeuwen, Phys. Rev. A 64, 023405 (2001).

[28] H.W. van der Hart, K. Burnett, Phys. Rev. A 62, 013407 (2000).

[29] Th. Weber, M. Weckenbrock, A. Staudte, L. Spielberger, O. Jagutzki, V. Mergel, F. Afaneh, G. Urbasch, M. Vollmer, H. Giessen, R. Dörner, J. Phys. B 33, L127 (2000). 
[30] R. Moshammer, B. Feuerstein, W. Schmitt, A. Dorn, C.D. Schröter, J. Ullrich, H. Rottke, C. Trump, M. Wittman, G. Korn, K. Hoffman, W. Sandner, Phys. Rev. Lett. 84, 447 (2000).

[31] Th. Weber, M. Weckenbrock, A. Staudte, L. Spielberger, O. Jagutzki, V. Mergel, F. Afaneh, G. Urbasch, M. Vollmer, H. Giessen, R. Dörner, Phys. Rev. Lett. 84, $443(2000)$.

[32] Th. Weber, H. Giessen, M. Weckenbrock, G. Urbasch, A. Staudte, L. Spielberger, O. Jagutzki, V. Mergel, M. Vollmer, R. Dörner, Nature 405, 658 (2000).

[33] B. Feuerstein, R. Moshammer, D. Fischer, A. Dorn, C.D. Schröter, J. Deipenwisch, J.R. Crespo Lopez-Urrutia, C. Höhr, P. Neumayer, J. Ullrich, H. Rottke, C. Trump, M. Wittmann, G. Korn, W. Sandner, Phys. Rev. Lett. 87, 043003 (2001).

[34] M. Weckenbrock, M. Hattas, A. Czasch, O. Jagutzki, L. Schmidt, T. Weber, H. Roskos, T. Löffler, M. Thomson, R. Dörner, J. Phys. B 34, L449 (2001).

[35] B. Witzel, N.A. Papadogiannis, D. Charalambidis, Phys. Rev. Lett. 85, 2268 (2000).

[36] R. Lafon, J.L. Chaloupka, B. Sheehy, P.M. Paul, P. Agostini, K.C. Kulander, L.F. DiMauro, Phys. Rev. Lett. 86, 2762 (2001).

[37] J.L. Chaloupka, R. Lafon, L.F. DiMauro, P. Agostini, K.C. Kulander, Opt. Express 8, 352 (2001).

[38] V. Stert, W. Radloff, C.P. Schulz, I.V. Hertel, Eur. Phys. J. D 5, 97 (1999).

[39] B. Walker, E. Mevel, B. Yang, P. Breger, J.P. Chambaret, A. Antonetti, L.F. DiMauro, P. Agostini, Phys. Rev. A 48, R894 (1993).

[40] O.V. Tikhonova, in: Super-Intense Laser-Atom Physics, Eds. B. Piraux, K. Rzazewski, Kluwer Academic Publishers, Dordrecht 2001, p. 85. 